\title{
Virtual Jewellery Shopping using Augmented Reality
}

\author{
Gaurav Salunke, Himanshu More, Rigved Shete, Prakash Kawade. \\ JSPM's Imperial College of Engineering and Research
}

\begin{abstract}
Building an object virtually 3D to use in a jewellery shops in which a virtual object will be placed instead of actual ornament in a real world using augmented reality. Using augmented reality, virtual object get superimpose on a real object at real time. In existing system user has to adjust himself/herself according to the object, where the position of object is fixed into the provided environment. Plus occlusion problem is the big threat of virtual object. By using ICP algorithm reconstruction of $3 \mathrm{D}$ object is possible. The object will be managed itself according to the user in real time by using HAAR algorithm by setting up the markers on the human face.
\end{abstract}

Keywords: Virtual Reality, Occlusion Problem, Augmented Reality, HAAR, ICP, jewellery shop application.

\section{INTRODUCTION}

Augmented reality is a technology that superimposed virtual object into real world. The jewellery shop application is the Augmented reality-based application in which user can wear the jewellery ornaments in real time.

The ornaments are precious, that's why it's important to take a good care of them. Customers used to handles the ornaments at the time of shopping, because of which ornaments can getting harmed or getting dirty and if they are not handle carefully might also be chances of breaking down. Here in this system the ICP algorithm is used for the 3D projection of the object for the inspection of the user before trial. HAAR algorithm is used to detect the customer's face and set the markers. The program takes the input as an image of the customer who is standing in front of screen which provides access to customer to see how they look in it. Thus, the system will help to customer for taking the decision of buying the ornament. This is achieved by using webcam.

System will detect and track the movement of the customer as soon as the customer selects a particular item to from application, that particular item will adjust itself onto that relevant face part of customer and hence the customer can try out various ornaments and decide whether that particular item suits him/her or not by them experience like manual shopping.

\section{A.RELATED WORK}

The augmented reality systems use the techniques based on perceptual and geometrical concepts for positioning the 3D objects [4]. Holo lenses are used by these techniques to avoid the position errors. Although the geometric technique is more efficient than perceptual, the movement of objects is not possible.

The object creation in virtual environment is a crucial part in the AR, for this the object reconstruction method is used for reproducing the material appearance of object [1] with inclusion of ICP algorithm. The system captures depth and colour images of object using RGBD camera. The reflectance of object is obtained by estimating the parameters of a reflectance model from the reconstructed shape and colour images.

The errors of the occlusion problem i.e. the difficulty in producing the virtual image of hollow or semi-transparent objects are addressed in [5]. These problems can be overcome by using the Vuforia environment.

Scene reconstruction has already been utilized for remote collaboration. However, the resolution of the resulting models was often too low. Thus, the range of potential applications was limited. World stabilized annotations such as drawings and place marks could only be used for simple instructions and view point of the user could vary only within a limited-range [2].

The study showed that in an interactive augmented reality setting, buyer's feelings of possession control and autotelic requirement for touch saliently impacted the relationship between striking recollections. The consequences of this review can help online merchants in utilizing enlarged reality intuitive innovation for building up purchaser influence model [3].

- In many previous works the human has to adjust himself such that he fits into the given image displayed in screen. This makes uncomfortable for user; the size of the humans may exceed the given frame on the screen.

- Hollow semi-transparent objects can't be constructed and presented in the AR environment properly.

\section{PROPOSED SYSTEM}

Our system will provide a virtual interaction to increase the security of expensive ornaments and will reduce the chance of damage of ornaments. Moreover the past similar methodologies suffered with the occlusion/semitransparent objects problems[2]. These problems are overcome by using the integrated closest point (ICP) algorithm for objects.

The model of virtual object is created by measuring shape and reflectance of real object using a RGB-D camera [1]. The RGB-D camera captures the depth images from different viewpoints to construct the 3D shape of the target object. Using the Iterative Closest Point (ICP) algorithm the point clouds are estimate the object motion with high accuracy.

The system calculates the correspondence between each vertex of reconstructed 3D model and coloured pixels in all 
frames by projecting the vertices of all camera viewpoints; it is determined which pixel in each colour image the vertex corresponds to. Hence by picking corresponding pixel values, intensity change at the vertex overall frame is obtained.

The HAAR algorithm is the face detection algorithm. It marks the hot points on the real image, this point can be then calculated and the virtual object is placed on the screen on corresponding points.

In the previous system the user had to adjust himself according to the frames to fit into the given images displayed on screen. This problem can be dealt by using the HAAR algorithm. This algorithm uses the AR markers for determination of the position of the virtual object on the screen.

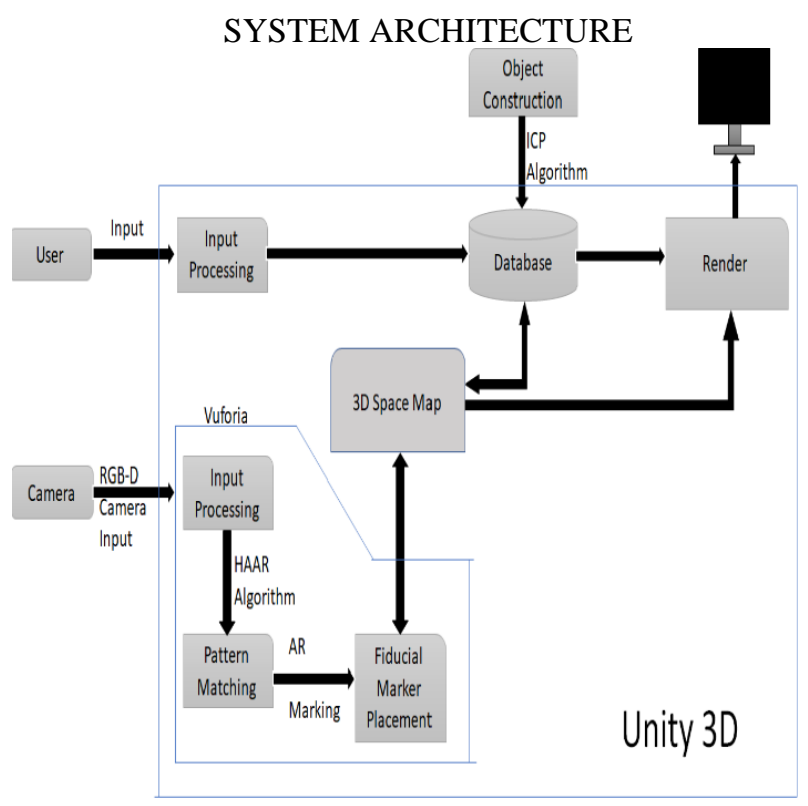

\section{ALGORITHMS}

\section{(A)ICP ALORITHM}

Step 1: For each point in the source point cloud, match the closest point in the reference point cloud.

Step 2: Estimate the combination of rotation and translation using a root mean square point to point distance metric minimization technique which will best align each source point to its match found in the previous step. This step may also involve weighting points and rejecting outliers prior to alignment.

Step 3: Transform the source points using the obtain transformation.

Step 4: Iterate (re-associate the points, and so on).

\section{(B)HAAR ALGORITHM}

Step1: Pick a pixel location from the image.

Step 2: Now crop a sub-image with the selected pixel as the centre from the source image with the same size as the convolution kernel.

Step 3: Calculate element wise product between the values of the kernel and the sub image.
Step 4: Add the result of the product.

Step 5: put the resultant value into the new image at the same place where you picked up the pixel location.

\section{RESULTS:}

This section is dedicated to discuss the results of the application. The performance of application was different for different AR environments.



The fig 1 shows the results of the application in which the virtual object (ring) is superimposed on real time feed of camera. This application is implemented using 'unity 3D' and 'vuforia' libraries with 'HAAR' algorithm for AR markings

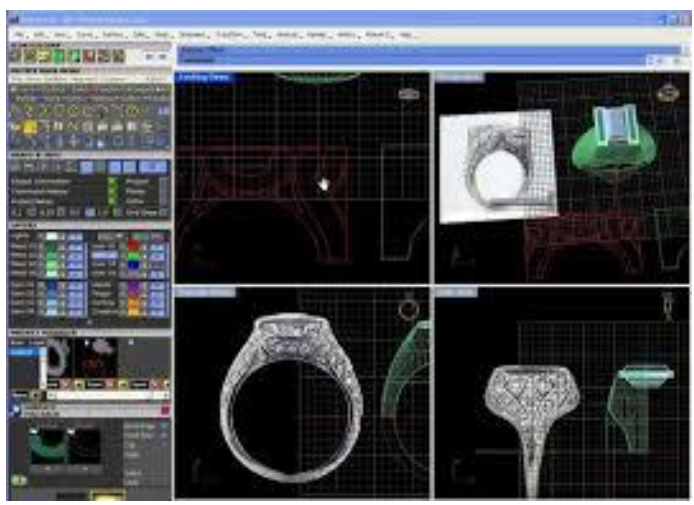

Fig.2 shows the construction of ring using 'Rhino' work base implementing ICP algorithm successfully handle occlusion problem.

\section{V.CONCLUSION}

In many previous works the human has to adjust himself such that he fits into the given image displayed in the screen. This makes uncomfortable for the user. Thus proposed system is more reliable than the previous existing systems. In this the human face is detected automatically so that the user may not fit in the given image.

This system could be used for enhancing security as it is developed in Augmented Reality. In the application user can view the ornaments in 3D which overcomes occlusion problem also. Hence, using the application user can wear the ornaments virtually, thus proposed system could be more reliable. This makes jewellery safe and moreover avoids the risk of theft. 


\section{REFERENCES}

[1] Seiji Tsunezaki, Ryota Nomura, Takashi Komuro and Norimichi Tsumura, "Reproducing Material Appearance of real Object using Mobile Augmented Reality", Tokyo, [2018].

[2] Jacob Ziollner, Erick Mendez and Daniel Wagner, "Augmented RealityRemote Collaboration with Dense Reconstruction", DAQRI Vienna [2018]

[3] Saikat Chakraborty and Deepak Gupta, "AStudy of the Factors Impacting the Adoption of Augmented Reality in Online Purchases in India",[2017].

[4] Wallace S. Lages, Yuan Li and Dowman, "Evolution of Environment-Independent Techniques for 3D Position Marking in Augmented Reality", Germany, [2018]

[5] D. P. Gadekar, N. P. Sable, A. H. Raut, "Exploring Data Security Scheme into Cloud Using Encryption Algorithms" International Journal of Recent Technology and Engineering (IJRTE), Published By:Blue Eyes Intelligence Engineering \& Sciences Publication, ISSN: 2277-3878, Volume-8 Issue-2, July2019, DOI: 10.35940/ijrte.B2504.078219, SCOPUS Journal.

[6] Sable NileshPopat*, Y. P. Singh," Efficient Research on the Relationship Standard Mining Calculations in Data Mining" in Journal of Advances in Science and Technology | Science \& Technology, Vol. 14, Issue No. 2, September-2017, ISSN 22309659.

[7] Sable NileshPopat*, Y. P. Singh," Analysis and Study on the Classifier Based Data Mining Methods" in Journal of Advances in Science and Technology | Science \& Technology, Vol. 14, Issue No. 2, September-2017, ISSN 2230-9659

[8] Jesus Gimeno, Sargio Casas, Cristina Portales and Marcos Fernandes,"Addressing the Occlusion Problem in Augmented Reality Environment with Phantom Hollow Object",Department of Computer Science University of Valencia, [2018]

[9] Mario Laurenze, Subastian Knopp and Phillipp Klimant, "Industrial Augmented reality: Requirement for an Augmented Reality Maintenance Worker Support System, Institute of Machine Tool and Production Process, Chemnitz University of Technology, [2018].

[10] Mayank Pandey, Dr. Manoj Wadhwa and Ms. Prabha Naik, "Tracking Algorithm for Augmented Reality System", International Journal of Engineering And Computer Science, [2014].

[11] Zhenliang Zhang, Dongdong Weng, Haiyan Jiang, Yue Liu and Yongtain Wang, "Inverse Augmented reality: A virtual Agent's Perspective", Beijing Institute of Technology, [2018].

[12] Prakash K. Ukhalkar, Dr. Rajesh N. Phursule , Dr Devendra P Gadekar, Dr Nilesh P Sable. (2020). Business Intelligence and Analytics: Challenges and Opportunities. International Journal of Advanced Science and Technology, 29(12s), 2669-2676.

[13] https://en.wikipedia.org/wiki/ Augmented_Reality. 